\title{
ALBUMINA E INDICE NEUTROFILO-LINFOCITO COMO PREDICTORES DE ESTADÍO TUMORAL EN PACIENTES CON CÁNCER GÁSTRICO
}

\author{
ALBUMIN AND NEUTROPHIL TO LYMPHOCYTE RATIO AS PREDICTORS OF TUMOR STAGE IN \\ PATIENTS WITH GASTRIC CANCER
}

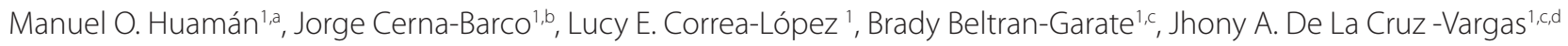

\begin{abstract}
RESUMEN
Introducción: El cáncer gástrico es un problema de salud pública a nivel mundial. Objetivo: Determinar la asociación entre albumina, marcadores inflamatorios y el estadío tumoral de los pacientes con cáncer gástrico en un Hospital Nacional del Perú. Métodos: Estudio de tipo observacional, analítico y retrospectivo correspondiente a los años 2017 y 2018. Se evaluaron las frecuencias y distribucion de las variables de estadío clínico según el AJCC 2018, albúmina como indicador de nutricion e Índice-Neutrófilo Linfocito (INL) como indicador de respuesta inflamatoria-inmunológica. Se consideró 2,44 como el punto de corte para INL elevado. Resultados: Fueron incluidos 96 pacientes. La edad promedio fue de 63,5 años $\pm 12,8$, la relacion hombre:mujer fue de $1: 1,80,2 \%$ provenían de la region de la costa del Perú; $70,8 \%$ tuvieron un estadío clínico avanzado y $85,4 \%$ correspondió a tamaño tumoral T3 y T4. El $64 \%$ presento grado histologico indeferenciado y un 30,1\% mostro evidencia de metastasis. La media de INL fue 2,94 $\pm 1,7$, y de albúmina fue $3,64 \mathrm{~g} / \mathrm{dl} \pm 0,6$, En el análisis bivariado se encontró una asociación significativa entre el nivel elevado de INL y el estadío clínico avanzado (OR: 4,46 IC 95\% 1,65-13,27 p<0,001), y entre los niveles bajos de albúmina sérica con estadio avanzado (OR: 13,02 IC 95\% 1,78-5,36 p<0,005). Conclusión: Se encontró un diagnóstico tardío en el $70 \%$ de los pacientes. El INL elevado como indicador de respuesta inflamatoria y la albúmina baja como indicador de nutricion son factores predictivos de estadio clínico avanzado en cáncer gástrico.
\end{abstract}

Palabras clave: Neoplasias gástricas, Estadificación de neoplasias, Índice neutrófilo-linfocito (fuente: DeCS BIREME).

\begin{abstract}
Introduction: Gastric cancer is a public health problem worldwide. Objective: To determine the association between albumin, inflammatory markers and tumor stage of gastric cancer patients in a National Hospital of Peru. Methods: An observational, analytical and retrospective study corresponding to the 2017 and 2018 years. The frequencies and distribution of the variables were evaluated: clinical stage according to the AJCC 2018, albumin as a nutrition indicator and Neutrophil Lymphocyte-Ratio (NLR) as an indicator of inflammatory and immune response. The value 2.44 was taken as the cut-off point for high NLR. Results: Were included 96 patients. The average age was 63.5 years \pm 12.8 , the male: female ratio was 1: 1, 80.2\% came from the region of the coast of Peru; $70.8 \%$ had an advanced clinical stage and $85.4 \%$ corresponded to tumor size T3 and T4. 64\% presented undifferentiated histological grade and $30.1 \%$ showed evidence of metastasis. The mean NLR was $2.94 \pm 1.7$, and albumin was $3.64 \mathrm{~g} / \mathrm{dl} \pm 0.6$. In the bivariate analysis, a significant association was found between the high level of NLR and the advanced clinical stage (OR: 4.46 $95 \% \mathrm{Cl} 1.65-13.27 \mathrm{p}<0.001)$, and between low levels of serum albumin with advanced stage (OR: 13.02 $95 \% \mathrm{Cl} 1.78-563.36 \mathrm{p}<0.005)$. Conclusion: A late diagnosis was found in $70 \%$ of the patients. High NLR as an indicator of inflammatory response and low albumin as an indicator of nutrition are predictors of advanced clinical stage in gastric cancer.
\end{abstract}

Key words: Stomach neoplasms; Neoplasm staging; Neutrophil-to-lymphocyte ratio (source: MeSH NLM).

\footnotetext{
${ }^{1}$ Instituto de Investigación en Ciencias Biomédicas, Facultad de Medicina Humana, Universidad Ricardo Palma, Lima-Perú.

2 Departamento de Cuidados Instensivos, Hospital Nacional Edgardo Rebagliatti Martí, Lima-Perú.

${ }^{3}$ Departamento de Oncologia Medica, Hospital Nacional Edgardo Rebagliatti Martí, Lima-Perú.

a Médico Cirujano, ${ }^{\text {b }}$ Médico Intensivista, ' Medico Oncólogo, d Doctor en Medicina.
}

Citar como: Manuel O. Huamán, Jorge Cerna-Barco, Lucy E. Correa-López, Brady Beltran-Garate, Jhony A. De La Cruz-Vargas. Albumina e indice neutrofilo-linfocito como factor predictor de estadío tumoral avanzado en pacientes con cáncer gástrico . Rev. Fac. Med. Hum. Abril 2020; 20(2):268-275. DOI 10.25176/RFMH.v20i2.2936 


\section{INTRODUCCIÓN}

El cáncer gástrico (CG) es un problema de salud pública ${ }^{(1,2)}$. Sigue siendo una causa importante de mortalidad por cáncer en el este de Asia, Europa del Este y América del Sur con más de 700,000 muertes anuales ${ }^{(3)}$. Más del $70 \%$ de los nuevos casos y muertes son de países en desarrollo, lo que resulta en una carga social importante ${ }^{(4)}$. Por lo tanto, el desarrollo de biomarcadores económicos y confiables para diagnosticar CG es crucial.

En USA y Europa, el carcinoma gástrico ha presentado un notable descenso en su incidencia ${ }^{(3)}$. No obstante, $y$ a pesar de las mejoras en los tratamientos quirúrgicos y oncológicos, la mortalidad de dicha neoplasia sigue siendo muy elevada, con unas tasas de supervivencia a los 5 años del diagnóstico de tan solo un 20-30\% en los países occidentales ${ }^{(3)}$. Dentro de este mal pronóstico general, se registra una notable variabilidad individual en cuanto a la supervivencia, por lo que es muy importante el poder conocer los factores predictivos acerca de la posible evolución de un determinado paciente ${ }^{(5)}$.

En el Perú, el CG, ocupa el primer lugar como causa de muerte prematura y se encuentra dentro de los cánceres mas frecuentes, con predominio en las regiones de Huncavelica, Huánuco, Pasco, Ayacucho, San Martin, Apurimac y Junín, relacionados con indicadores de pobreza ${ }^{(6-8)}$.

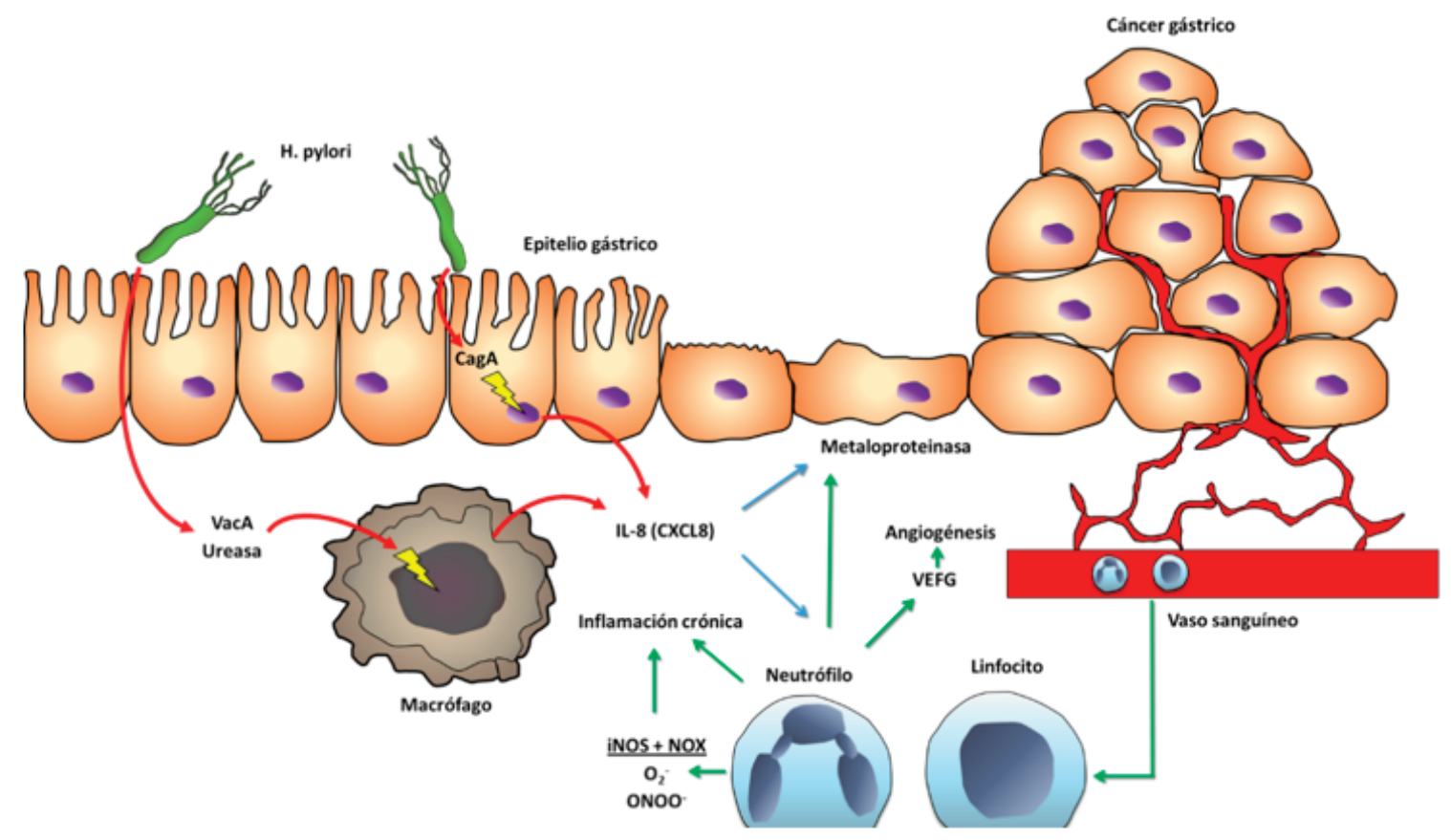

Figura 1. Papel de la respuesta inmune en la progresión del cáncer gástrico. Adaptado de: Gobert AP, Wilson KT. Human and Helicobacter pylori Interactions Determine the Outcome of Gastric Diseases. Curr Top Microbiol Immunol.

Se han identificado como principales factores de riesgo la infección por Helicobacter pylori, el bajo nivel socioeconómico, dieta alta en carnes rojas, sedentarismo y predisposición genética(6,9-11).

La inflamación parece ser el telon de fondo para el desarrollo y la progresión tumoral, ya sea en la sangre o en el tejido tumoral. La relación entre neutrófilos y linfocitos conocido como Índice Neutrófilo-Linfocito (INL) se ha considerado un indicador de inflamación sistémica en muchos estudios clínicos actuales, incluyendo el cáncer gástrico ${ }^{(12-24)}$, en ausencia de infección obvia ${ }^{(25)}$.

La inflamación crónica mediada principalmente por una activación prolongada de neutrófilos favorece la carcinogénesis a través de la genotoxicidad, inhibición de las células $T$, angiogénesis e invasión (ver Figura1). De manera contraria la población linfocítica posee una función antitumoral al reconocer las células tumorales y activar la actividad citolítica tumoral a través de las células NK y TCD8 $(+)^{(26)}$.

Si bien el tamaño tumoral, el grado de invasión, el compromiso ganglionary la presencia de metástasis han sido utilizados para el estadiaje tumoral y pronostican el desarrollo y agresividad de la neoplasia, existe mucha variabilidad en la sobrevida de los pacientes que se encuentran en un mismo estadío, lo cual sugiere la existencia de factores adicionales que influencian el comportamiento tumoral. Se ha propuesto en diversos estudios la división de los estadios I y II como estadios localizados y III y IV como estadios avanzados ${ }^{(27,28)}$. La 
albumina como un indicador del estado nutricional, la capacidad de respuesta a los tratamientos y complicaciones ha sido mencionado en múltiples estudios.

La utilidad del INL y la albumina para ayudar a clasificar los estadios tumorales tempranos de los avanzados es un tema aun en controversia. por lo que el objetivo de este estudio fue evaluar la utilidad del INL y la albumina como factores predictivos de estadio clínico en pacientes con diagnóstico de cáncer gástrico.

\section{MÉTODOS}

\section{Diseño y población}

Se realizó un estudio observacional, analítico, de tipo retrospectivo, en el que se incluyeron a todos los pacientes hospitalizados con diagnóstico clínico de adenocarcinoma gástrico durante el periodo julio 2017 y julio 2018 en el servicio de cirugía de estómago del hospital nacional Edgardo Rebagliati Martins (HNERM) de la seguridad social del Perú (ESSALUD).

Se excluyeron a los pacientes con datos incompletos en el preoperatorio (data insuficiente del archivo del servicio de cirugía de estómago) y a aquellos que durante el acto operatorio se determinó que eran tumores de la unión esófago-gástrica.

\section{Variables de estudio}

Dentro de las variables incluidas en el estudio se tomó en cuenta variables sociodemográficas-clínicas (sexo, edad, lugar de procedencia) laboratoriales (Índice NeutrófiloLinfocito, Albumina) y el estadio clínico tumoral según el TNM para cáncer gástrico de la AJCC 2018.

El INL se obtuvo como el resultado del cociente del número de neutrófilos entre linfocitos encontrados en hemograma y la albumina del examen bioquímico tomado preoperatoriamente. Se consideró INL elevado a todo valor mayor igual a 2,44 basándonos en estudios realizados previamente en Latinoamérica.

\section{Análisis estadístico}

La recolección de datos se realizó mediante la ficha de recolección La digitalización de los datos se realizó en el programa Excel 8.0 y el análisis con STATA 12.0. Se realizó estadística descriptiva con medidas de tendencia central y dispersión. Para estadística inferencial se calculó como medida de asociación OR, con IC95\% con un nivel de significancia estadística $<0,05$. Las variables categóricas se analizaron con estadísticos de chi cuadrado, fisher y $t$ de estudent para variables cuantitativas.

\section{Criterios éticos}

El estudio fue aprobado por el Comité de ética en
Investigación del hospital y se desarrollo en el curso de tesis del Instituto de Investigación en Ciencias Biomédicas, según metodología publicada(29).

\section{RESULTADOS}

Durante el período julio de 2017 a julio de 2018, se incluyeron inicialmente a 134 pacientes hospitalizados en el servicio de cirugía de estomago del HNERM con diagnóstico de cáncer gástrico. Se incluyeron en el análisis preliminar los datos de 98 pacientes que cumplieron con los criterios de inclusión del trabajo. Se identificaron 2 pacientes con datos atípicos de INL de acuerdo al método de Tukey, motivo por el que se les excluyó. Finalmente se analizaron los datos de 96 pacientes (ver Figura 1).

La edad promedio fue de 63,5 años $\pm 12,8$, el $68,7 \%$ de los pacientes se encontraban entre los 45 y 75 años. Se determinó que la edad seguía una distribución normal. En la distribución de los pacientes según sexo, el más frecuente fue el femenino, representado un 52\% de la muestra total, conservando una relación de 1:1. En cuanto al lugar de procedencia se evidencia que la mayoría de pacientes vivían en la región de la costa del Perú, representando el 80,2\% de la muestra.

Con respecto al estadio clínico $70.8 \%$ tuvieron un estadio avanzado (cTNM III-IV), en cuanto al criterio tumoral se evidenció que la mayoría de pacientes se diagnosticaron con tamaño tumoral avanzado en 85.4\%, encontrando ganglios positivos en el 57,2\%; en cuanto al criterio de metástasis fue positivo en el $30,2 \%$ de pacientes (ver Tabla 1).

En el análisis del Índice Neutrófilo-Linfocito (INL) la media fue $2,94 \pm 1.7$ y la mediana 2,44 (RIC 1,68-3,84) asimismo se observó que presentaba una distribución no paramétrica, confirmada por test de Shapiro-Wilk $(p<0,001)$. Según el estadío clínico (cTNM), en los estadíos localizados (cTNM I-II) la media fue 2,13 \pm 0,9, la mediana 1.97 (RIC 1,45-2,63), mientras que en los estadios avanzados (cTNM III-IV) la media fue 3,28 $\pm 1,8$, la mediana 2.73 (RIC 1,79-4,35) (ver Figura 3). Al comparar ambas medias con el estadístico t-student se obtuvo una diferencia estadísticamente significativa ( $p<0,003$ ). La media de albumina fue de 3,64 g/dl $\pm 0,6$ en el grupo de estadio avanzado fue de 3,51 g/dl $\pm 0,6$

En la Tabla 2 se describen las características clínicolaboratoriales de los pacientes con cáncer gástrico según el estadío clínico. Se analizaron las variables de acuerdo a su naturaleza con los estadísticos t-student, chi2 y prueba exacta de Fisher para determinar el valor p. En el estadio clínico (cTNM) se evidencia una mayor proporción de pacientes con estadíos avanzados con INL elevado (0.94:1) que en aquellos con INL normal 
$(0,20: 1)(p<0,001)$. El resto de variables no demostraron tener diferencias estadísticamente significativas.

Se obtuvo un OR de 4,64 (IC95\% 1,65-13,27) entre el INL elevado y el estadio clínico avanzado el cual fue estadísticamente significativo $(p=0,001)$, de manera similar con los niveles bajos de albumina sérica OR $13,02$ IC95\% 1,78-5,36 ( $p=0,003)$.

Pacientes hospitalizados con giagnóstico de cáncer gástrico en el servicio de cirugía de estomago del HNERM durante los años 2017-2018

$\Sigma=134[100 \%]$

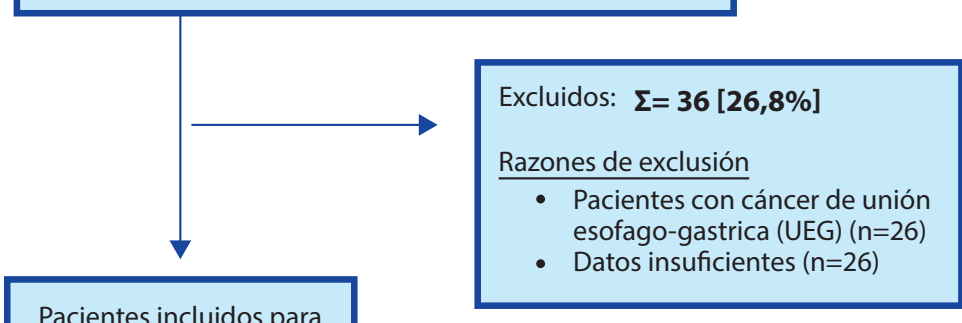

Pacientes incluidos para

el análisis preeliminar $\mathrm{n}=98$

Excluidos: $\mathbf{\Sigma}=\mathbf{2}[\mathbf{1}, \mathbf{4} \%]$

Razones de exclusión

- Valores atípicos de INL $(n=2)$

Pacientes incluidos para

el análisis final

$\mathrm{n}=96$

Figura 2. Flujograma de selección de la muestra.

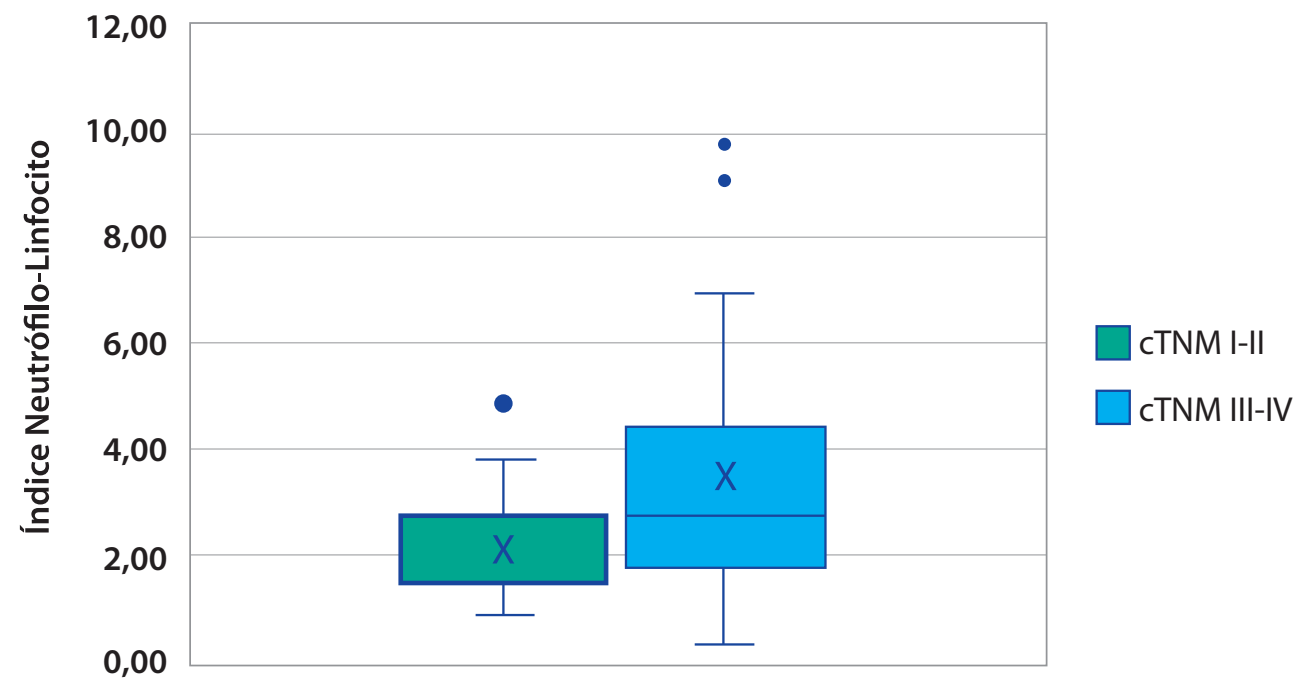

Estadío clínico (cTNM)

Figura 3. Distribución de valores de Índice Neutrófilo-Linfocito (INL) según estadío clínico. 
Tabla 1. Distribución de los pacientes según edad, sexo, lugar de procedencia, criterio tumoral, criterio ganglionar, criterio de metástasis y estadío clínico.

\section{Edad (años)}

Media ( \pm SD)

63,5

$( \pm 12,8)$

\section{Sexo}

Masculino

Femenino

\section{Lugar de procedencia}

Costa

Sierra

cT

cM

$\mathrm{N}$ negativo

67

$(69,8 \%)$

$N$ positivo

\section{CTNM}

I-II

III-IV

Tabla 2. Análisis bivariado de las características clínico-laboratoriales en pacientes con cáncer gástrico según el estadio clínico.

\begin{tabular}{|c|c|c|c|c|c|c|}
\hline Variables & $\begin{array}{c}\text { cTNM III-IV } \\
n=68 \\
(70,8 \%)\end{array}$ & $\begin{array}{c}\text { cTNM I-II } \\
n=28 \\
(20,1 \%)\end{array}$ & OR & IC $95 \%$ & chi2 & $\mathbf{p}$ \\
\hline Edad & & & 0,75 & $0,27-1,98$ & 0,41 & 0,52 \\
\hline$<65$ & $34(35,4 \%)$ & $12(12,5 \%)$ & & & & \\
\hline$\geq 65$ & $34(35,4 \%)$ & $16(16,6 \%)$ & & & & \\
\hline Sexo & & & 1,08 & $0,41-2,89$ & 0,04 & 0,85 \\
\hline Masculino & $33(34,8 \%)$ & $13(13,5 \%)$ & & & & \\
\hline Femenino & $35(36,4 \%)$ & $15(15,3 \%)$ & & & & \\
\hline INL & & & 4,46 & $1,65-13,27$ & 11,06 & 0,0009 \\
\hline$\geq 2,44$ & $49(51,0 \%)$ & $10(10,4 \%)$ & & & & \\
\hline$<2,44$ & $19(19,7 \%)$ & $18(18,7 \%)$ & & & & \\
\hline Albúmina sérica & & & 13,02 & $1,78-5,36$ & 8,79 & 0,0030 \\
\hline$<3,5$ & $24(30,3 \%)$ & $1(1,2 \%)$ & & & & \\
\hline$\geq 3,5$ & $35(44,3 \%)$ & $19(24,0 \%)$ & & & & \\
\hline
\end{tabular}




\section{DISCUSIÓN}

Nuestro estudio encontró que tanto el INL elevado, como marcador de respuesta inflamatoria e inmunológica, y la albúmina baja, como indicador de nutrición, fueron factores predictivos de estadio clínico avanzado en cáncer gástrico.

El cáncer gástrico es un problema de salud pública, debido a su prevalencia, agresividad y diagnóstico tardío. Es necesario desarrollar métodos para su detección oportuna, y manejo estratificado ${ }^{(8)}$. Múltiples variables que influyen sobre el pronóstico dependen del estado general del paciente, estado nutricional, edad, comorbilidades, de las características histológicas del tumor, del grado de crecimiento y de la extensión de la neoplasia, así como de la respuesta inflamatoria sistémica del paciente frente al tumor ${ }^{(6,10,17)}$. Dicha respuesta inflamatoria está relacionada con el crecimiento y la capacidad de invasión y de metástasis de la neoplasia ${ }^{(18)}$.

La respuesta inflamatoria sistémica acompaña el desarrollo del cáncer, tanto para el cáncer temprano o avanzado, lo que nos proporciona nuevos métodos para la identificación temprana de $\mathrm{CG}^{(30,31)}$. Estudios anteriores respaldan la hipótesis de la "transformación de inflamación y cáncer"(32-34): la gastritis crónica puede causar $\mathrm{CG}^{(32)}$; La hepatitis viral crónica puede provocar cirrosis, que a su vez conduce a cáncer de hígado ${ }^{(33)}$. El epitelio del tracto gastrointestinal está continuamente expuesto al ambiente externo y es susceptible a la inflamación debido a varios patógenos u otros estímulos ${ }^{(34)}$.

Diversos indicadores inflamatorios sobre el pronostico en tumores del tubo digestivo, páncreas e hígado han sido evaluados, fundado en la relación entre respuesta inflamatoria y la progresión del tumor ${ }^{(11)}$. Destacan el índice de Glasgow basado en los valores de la proteína C reactiva y la albúmina ${ }^{(19)}$, el cociente neutrófilos/ linfocitos circulantes ${ }^{(20)}$, indice plaquetas/linfocitos ${ }^{(21)} y$ plaquetas/neutrofilos, también denominado índice de Onodera ${ }^{(22)}$.

La resecabilidad es un criterio que se confirma tras la cirugía y que en el momento del diagnóstico tan solo puede presumirse mediante la clasificación tumoral TNM clínica, cuya precisión es limitada y tiende a la infra-estadificación en al menos un $20 \%$ de los $\operatorname{casos}^{(9,10)}$. Por todo ello, precisamos disponer de otros parámetros predictivos, independientes del TNM clínico, y que se puedan determinar en el momento del diagnóstico del tumor ${ }^{(5,6)}$.

En el presente estudio se observó que la mayoría de pacientes se encontraban entre los 60-75 años con una edad promedio de 63,5 años $\pm 12,8$, asimismo el $62,5 \%$ era mayor de 60 años. No se encontró una asociación significativa entre la edad avanzada y el estadío clínico avanzado. Esto concuerda con estudios realizados en Brasil por Szor et al. ${ }^{(34)}$, sin embargo, en el análisis multivariado se encontró que la edad mayor a 65 podía tener cierta influencia en el pronóstico de los pacientes con cáncer gástrico.

La proporción de la muestra de acuerdo a sexo fue de 1:1. No se encontró asociación entre el sexo y el INL elevado, albúmina sérica baja y estadío tumoral avanzado. Si bien el sexo femenino se ha vinculado con un efecto protector antitumoral al interaccionar con los receptores de estrógenos, esto no ha sido demostrado definitivamente en metaanálisis y estudios aleatorizados ${ }^{(35)}$.

La mayor proporción de pacientes (80,2\%) procedían de la zona de la costa. Según el Ministerio de Salud la mayor incidencia de pacientes se encuentra en la costa ${ }^{(8)}$, esto se correlaciona con nuestros hallazgos. Esta situación esta relacionada con la densidad poblacional en la región de la costa del Perú y las corrientes migratorias internas.

De los pacientes hospitalizados por cáncer gástrico 7 de cada 10 correspondían a estadíos clínicos avanzados, esto refleja la escasa presencia de prevención primaria y secundaria en nuestro sistema de salud en cuanto a una patología que afecta notablemente a nuestra población. De manera similar la mayoría de pacientes tuvieron criterio tumoral avanzado en el $84,5 \%$, criterio ganglionar positivo en el 57,3\% y criterio de metástasis positivo en el $30,2 \%$.

Es necesario fortalecer el primer nivel de atención y mejorar las actividades preventivo promocionales con el fin de disminuir la prevalencia y aumentar la proporción de pacientes diagnosticados en estadíos tempranos ${ }^{(7)}$, haciendo énfasis en Medicina del Estilo de Vida.

La relación entre la inflamación sistémica y los tumores ha sido de interés para los investigadores. En 1869, Ashworth propuso por primera vez el concepto de "Celulas Tumorales Circulantes" CTC. Después de años de investigación, las pruebas de CTC se aprobaron para uso clínico. Sin embargo, la rareza, la heterogeneidad y el alto costo de las pruebas de CTC plantean desafíos para usarlas como biomarcadores ${ }^{(35-37)}$. Esto ha llevado a los investigadores a considerar seriamente el fenómeno de que las células cancerosas ingresan a la sangre periférica en la etapa inicial del cáncer, pero la metástasis temprana es rara $^{(38,39)}$. Publicaciones recientes han informado que las CTC que ingresan a la sangre periférica desencadenan una respuesta inmune que incluye un aumento en la proporción de macrófagos y neutrófilos asociados con el cáncer. Li(40) observó un aumento en la proporción de neutrófilos de sangre periférica en la progresión de tumores malignos, lo que se asoció con el pronóstico. Burr ${ }^{(41)}$ señaló que 
los medicamentos antiinflamatorios no esteroideos pueden reducir el riesgo de inflamación sistémica y tumorigénesis.

El punto de corte para determinar el valor elevado del INL es motivo de debate en la actualidad. Como marcador inflamatorio es utilizado en diferentes patologías, el cáncer gástrico es una de ellas. En 1976 Nakahara et al. acuñaron el término INL en la literatura médica y determinó su asociación con el pronóstico de pacientes con cáncer de pulmón. El punto de corte ha sido definido a través de curvas ROC de manera que puedan ser identificados fácilmente aquellos pacientes con mal pronóstico ${ }^{(28)}$. Según Min et al. el punto de corte promedio seria de 3,00. Deng et al de acuerdo a su análisis encontró que el punto de corte adecuado sería 2,46 . Sin embargo, Szor et al en 383 pacientes de origen brasileño determinaron que el punto de corte adecuado sería 2,44 siendo este el punto de corte utilizado en este estudio. La medida del INL no está estandarizada por lo cual se requieren mayor cantidad de estudios en diferentes poblaciones latinas con cáncer gástrico.

En nuestro estudio el promedio de INL fue 2,94 $\pm 1,7$ el $61,5 \%$ de los pacientes tenía un valor de INL elevado, en particular el grupo con estadio clínico avanzado $(72,0 \%)$ con una media de 3,28 $\pm 1,8$ teniendo este valor diferencia estadísticamente significativa con la media del grupo con estadio localizado $(p<0,003)$.

En el análisis bivariado se encontró que los pacientes con INL elevado tenían 4 veces más riesgo de presentar estadío clínico avanzado. Asimismo, Rodríguez C. en un hospital de Cajamarca encontró un aumento en la probabilidad de tener un estadío clínico avanzado en 6 veces, de manera similar en China, Min et al. demostraron un aumento en el riesgo de 1,5 veces.

Los neutrófilos constituyen el $50-70 \%$ de todos los glóbulos blancos en la circulación humana con un promedio de vida de 5.4 días en la condición homeostática de respuesta al estrés oxidativo ${ }^{(42)}$. Actualmente se cree que promueven la proliferación, vascularización y metástasis de las células cancerosas al producir quimiocinas proangiogénicas y factor de crecimiento endotelial vascular ${ }^{(43,44)}$. Actualmente se cree que los linfocitos en sangre periférica causan citotoxicidad sinérgica y ejercen propiedades supresoras de tumores ${ }^{(45)}$.

De manera interesante un trabajo recientemente publicado por Tiangy Fang y col $^{(46)}$, mostro que los marcadores inflamatorios sistémicos INL y IPL eran más valiosos para el diagnóstico de CG que los marcadores tumorales tradicionales CEA y CA19-9, dicho valor diagnóstico fue más alto en pacientes masculinos que femeninos con CG.

Ademas, el INL fue significativamente mayor en pacientes con CG que en sujetos normales, lo que también indicó que la respuesta inflamatoria del tumor basada en neutrófilos en la sangre periférica de los pacientes con tumor fue más fuerte que el efecto inmunitario antitumoral basado en linfocitos ${ }^{(45)}$.

En nuestro estudio, la albúmina fue el único parámetro bioquímico que presentó significancia estadística ( $p=$ $0,003)$ que se relaciona como predictor de enfermedad avanzada en nuestra población.

Numerosos biomarcadores han sido propuestos en cáncer gástrico. El mayor aporte de nuestro trabajo es que la albumina como marcador del estado nutricional del paciente con cáncer gástrico y el INL como indicador inflamatorio y de respuesta inmunológica, constituyen dos parametros simples, de fácil acceso y de muy bajo costo, con capacidad de predecir el estadio clínico en pacientes con cáncer gástrico. Nuestro análisis representaría uno de los primeros reportes en población peruana que menciona la hipoalbuminemia y el INL asociados a estadio avanzado en pacientes con cáncer gástrico.

Como otros trabajos publicados, el nuestro presenta la limitación de no ser prospectivo, lo que podría reducir la exactitud de los datos recogidos en la historia clínica. Corresponde a un estudio unicentrico, con limitado tamaño de la muestra.

Futuros estudios, de naturaleza prospectiva y multicentrica, que incluyan la evaluación del INL y la albumina como factores pronósticos de supervivencia global y supervivencia libre de enfermedad son necesarios.

\section{CONCLUSIÓN}

Se encontró un diagnóstico tardío en el 70\% de los pacientes. El INL como indicador de respuesta inflamatoria y la albúmina como indicador de nutrición son factores predictivos de estadio clínico avanzado en cáncer gástrico.

Contribuciones de autoría: Los autores participaron en la génesis de la idea, diseño de proyecto, recolección e interpretación de datos, análisis de resultados y preparación del manuscrito del presente trabajo de investigación.

\section{Financiamiento: Autofinanciado.}

Conflicto de interés: Los autores declaran no tener conflicto de interés en la publicación de este artículo.

Recibido: 16 de diciembre de 2019

Aprobado: 30 de marzo de 2020

Correspondencia: Manuel Huaman Garcia.

Dirección: INICIB, Facultad de Medicina Humana, Pabellón J, Edificio Administrativo, 2do piso. Avenida Benavides 5440, Surco, Lima - Perú.

Teléfono: 987408269

Correo: manuelhuamangarcia1@gmail.com 


\section{REFERENCIAS BIBLIOGRÁFICAS}

1. Bray, F., Ferlay, J., Soerjomataram, l., Siegel, RL, Torre, LA y Jemal, A. (2018), Estadísticas globales de cáncer 2018: Estimaciones de incidencia y mortalidad de GLOBOCAN en todo el mundo para 36 cánceres en 185 países. CA: A Cancer Journal for Clinicians, 68: 394-424. doi: 10.3322 / caac.21492

2. The Lancet. GLOBOCAN 2018: counting the toll of cancer,The Lancet, Volume 392, Issue 10152, 985. https://doi.org/10.1016/S0140-6736(18)32252-9

3. RL Siegel, KD Miller y A. Jemal, "Estadísticas de cáncer, 2018", CA: a Cancer Journal for Clinicians, vol. 68, no. 1, págs. 7-30, 2018.Ver en: Sitio del editor | Google Académico.

4. Global Burden of Disease Cancer Collaboration, "Incidencia global, regional y nacional del cáncer, mortalidad, años de vida perdidos, años vividos con discapacidad y años de vida ajustados por discapacidad para 32 grupos de cáncer, 1990 a 2015: un análisis sistemático para el estudio de la carga global de enfermedad, " JAMA Oncology, vol. 3, no. 4, págs. 524-548, 2017.Ver en: Sitio del editor | Google Académico.

5. Van Cutsem E, Sagaert X, Topal B, Haustermans K, Prenen H. Gastric cancer. Lancet Lond Engl. 26 de 2016;388(10060):2654-64.

6. Ramos W, Guerrero N, Medina J, Guerrero PC. Análisis de la situación del cánce en el Perú, 2018. Lima: Centro Nacional de Epidemiología, Prevención y Control de Enfermedades; 2019.

7. Torres-Román JS, Grados-Sánchez O. Cáncer gástrico en el Perú: una realidad susceptibilidad de cambio. Rev. gastroenterol. Perú [Internet]. 2015 Jul [citado 2019 Feb 05]; 35(3): 276-276. Disponible en: http://www.scielo.org.pe/scielo. php?script=sci_arttext\&pid=S1022-51292015000300013\&lng=es

8. Ministerio de Salud. Analisis de la situacion del cancer en el Peru, 2013. Lima: Ministerio de Salud; 2013. Disponible en: http://www.dge.gob.pe/portal/docs/ asis_cancer.pdf

9. de Martel C, Forman D, Plummer M. Gastric cancer: epidemiology and risk factors Gastroenterol Clin North Am. junio de 2013;42(2):219-40.

10. Karimi P, Islami F, Anandasabapathy S, Freedman ND, Kamangar F. Gastric Cancer: Descriptive Epidemiology, Risk Factors, Screening, and Prevention. Cance Epidemiol Biomark Prev Publ Am Assoc Cancer Res Cosponsored Am Soc Prev Oncol. mayo de 2014;23(5):700-13.

11. Kelley JR, Duggan JM. Gastric cancer epidemiology and risk factors. J Clin Epidemiol. enero de 2003;56(1):1-9.

12. Ayala de la Peña F, Yufera Soler JC, Ivars MA, Fernandez Sanchez A, Navarro Manzano E, De la Morena Barrio P, et al. PNeutrophil-lymphocyte ratio (NLR) as a prognostic factor in metastatic breast cancer. Ann Oncol [Internet]. el 1 de septiembre de 2017 [citado el 12 de junio de 2018];28(suppl_5). Disponible en: http://academic.oup.com/annonc/article/doi/10.1093/annonc/mdx365.046/41085 12/283PNeutrophillymphocyte-ratio-NLR-as-a-prognostic

13. Azab B, Camacho-Rivera M, Taioli E. Average Values and Racial Differences of Neutrophil Lymphocyte Ratio among a Nationally Representative Sample of United States Subjects. PLOS ONE. el 6 de noviembre de 2014;9(11):e112361.

14. Chen $Y$, Yan H, Wang $Y$, Shi $Y$, Dai G. Significance of baseline and change in neutrophil-to-lymphocyte ratio in predicting prognosis: a retrospective analysis in advanced pancreatic ductal adenocarcinoma. Sci Rep. el 9 de abril de 2017:7(1):753. 15. Huang Q, Zhou L, Zeng W, Ma Q, Wang W, Zhong M, et al. Prognostic Significance of Neutrophil-to-Lymphocyte Ratio in Ovarian Cancer: A Systematic Review and Meta-Analysis of Observational Studies. Cell Physiol Biochem. 2017:41(6):2411-8.

16. Lalani A-KA, Xie W, Martini DJ, Steinharter JA, Norton CK, Krajewski KM, et al. Change in neutrophil-to-lymphocyte ratio (NLR) in response to immune checkpoin blockade for metastatic renal cell carcinoma. J Immunother Cancer. el 22 de enero de 2018:6:5.

17. Orditura M, Galizia G, Diana A, Saccone C, Cobellis L, Ventriglia J, et al. Neutrophi to lymphocyte ratio (NLR) for prediction of distant metastasis-free survival (DMFS) in early breast cancer: a propensity score-matched analysis. ESMO Open. el 1 de marzo de 2016;1 (2):e000038.

18. Ou Q, Cheng J, Zhang L, Wang H, Wang W, Ma Y. The prognostic value of pretreatment neutrophil-to-lymphocyte ratio in breast cancer: Deleterious or advantageous? Tumor Biol. el 1 de junio de 2017;39(6):1010428317706214.

19. Ozyurek BA, Ozdemirel TS, Ozden SB, Erdogan Y, Kaplan B, Kaplan T. Prognostic Value of the Neutrophil to Lymphocyte Ratio (NLR) in Lung Cancer Cases. Asian Pac J Cancer Prev APJCP. 2017;18(5):1417-21.

20. Tang X, Du P, Yang Y. The clinical use of neutrophil-to-lymphocyte ratio in bladder cancer patients: a systematic review and meta-analysis. Int J Clin Oncol. el 1 de octubre de 2017:22(5):817-25.

21. Urrejola Gl, Bambs CE, Espinoza MA, Gellona J, Zúñiga ÁM, Molina ME, et al. Un índice neutrófilo/linfocito elevado se asocia a peor pronóstico en cáncer de colon etapa II resecado. Rev Médica Chile. mayo de 2013;141(5):602-8.

22. Wang J, Jia Y, Wang N, Zhang X, Tan B, Zhang G, et al. The clinical significance of tumor-infiltrating neutrophils and neutrophil-to-CD8+ lymphocyte ratio in patients with resectable esophageal squamous cell carcinoma. J Transl Med. el 7 de enero de 2014;12:7.

23. Xue $P$, Kanai $M$, Mori $Y$, Nishimura $T$, Uza N, Kodama $Y$, et al. Neutrophil-tolymphocyte ratio for predicting palliative chemotherapy outcomes in advanced pancreatic cancer patients. Cancer Med. abril de 2014;3(2):406-15.

24. Beltrán BE, Castro D, De La Cruz-Vargas JA, Cotrina E, Gallo A, Sotomayor EM, et al. The neutrophil-lymphocyte ratio is prognostic in patients with early stage aggressive peripheral T cell lymphoma. Br J Haematol. febrero de 2019;184(4):650-3
25. Szor DJ, Roncon Dias A, Pereira MA, Ramos MFKP, Zilberstein B, Cecconello I, et al. Neutrophil-lymphocyte ratio is associated with prognosis in patients who underwent potentially curative resection for gastric cancer. J Surg Oncol. abril de 2018;117(5):851-7.

26. He Q, Li G, Ji X, Ma L, Wang X, Li Y, et al. Impact of the immune cell population in peripheral blood on response and survival in patients receiving neoadjuvant chemotherapy for advanced gastric cancer. Tumor Biol. el 1 de mayo de 2017;39(5):1010428317697571.

27 NY Kim, D.-H. Chun, SY Kim et al., "Valor pronóstico de los índices inflamatorios sistémicos, NLR, PLR y MPV, para predecir la supervivencia a 1 año de pacientes sometidos a cirugía citorreductora con HIPEC", Journal of Clinical Medicine, vol. 8, no. 5, p. 589, 2019.Ver en: sitio del editor | Google Académico

28. Deng Q, He B, Liu X, Yue J, Ying H, Pan Y, et al. Prognostic value of pre-operative inflammatory response biomarkers in gastric cancer patients and the construction of a predictive model. J Transl Med [Internet]. el 18 de febrero de 2015 [citado el 14 de enero de 2019];13. Disponible en: https://www.ncbi.nlm.nih.gov/pmc/articles/ PMC4343078/

29. Jhony A. De La Cruz-Vargas, Lucy E. Correa Lopez, Maria del Socorro Alatrista Gutierrez de Bambaren, Hector Sanchez Carlessi, Consuelo Luna Muñoz, Maria Loo Valverde, et all. Promoting research in medical students and increasing scientific production in universities: Experience of the Undergraduate Thesis Workshop Course. Educacion Medica, Volume 20, Issue 4, July-August 2019, Pages 199-205. https://doi.org/10.1016/j.edumed.2018.06.003

30. CI Diakos, KA Charles, DC McMillan y SJ Clarke, "Inflamación relacionada con e cáncer y efectividad del tratamiento", The Lancet Oncology, vol. 15, no. 11, págs. E493 - e503, 2014.Ver en: sitio del editor | Google Académico

31. RD Dolan, BJA Laird, PG Horgan y D. McMillan, "El valor pronóstico de la respuesta inflamatoria sistémica en ensayos clínicos aleatorizados en cáncer: una revisión sistemática", Critical Reviews in Oncology / Hematology , vol. 132, págs. 130-137, 2018.Ver en: sitio del editor | Google Académico

32. RD Dolan, ST McSorley, PG Horgan, B. Laird y DC McMillan, "El papel de la respuesta inflamatoria sistémica en la predicción de resultados en pacientes con cáncer inoperable avanzado: revisión sistemática y metaanálisis ", Critical Reviews in Oncology / Hematology, vol. 116, pp. 134-146, 2017.Ver en: sitio del editor | Google Académico

33. H. Kuper, W. Ye, U. Broomé et al., "El riesgo de cáncer de hígado y de vías biliares en pacientes con hepatitis viral crónica, alcoholismo o cirrosis" , Hepatology, vol. 34 no. 4, págs. 714-718, 2001.Ver en: Sitio del editor | Google Académico

34. J. Candido y T. Hagemann, "Inflamación relacionada con el cáncer", Journal of Clinical Immunology, vol. 33, Suplemento 1, págs. 79-84, 2013.Ver en: Sitio del editor | Google Académico

35. T. Wu, B. Cheng y L. Fu, "Aplicaciones clínicas de las células tumorales circulantes en farmacoterapia: desafíos y perspectivas", Molecular Pharmacology, vol. 92, no. 3, págs. 232-239, 2017.Ver en: Sitio del editor | Google Académico

36. Z. Shen, A. Wu y X. Chen, "Tecnologías de detección actuales para las células tumorales circulantes", Chemical Society Reviews , vol. 46, no. 8, págs. 2038-2056, 2017.Ver en: sitio del editor | Google Académico

37. Z. Zhu, S. Qiu, K. Shao e Y. Hou, "Progreso y desafíos de secuenciar y analizar las células tumorales circulantes", Cell Biology and Toxicology , vol. 34, no. 5, págs. 405-415, 2018.Ver en: Sitio del editor | Google Académico

38. A. Dasgupta, AR Lim y CM Ghajar, "Células tumorales circulantes y diseminadas: ¿heraldos o iniciadores de metástasis?" Oncología Molecular, vol. 11, no. 1, págs. 40-61, 2017.Ver en: Sitio del editor | Google Académico

39. DS Micalizzi, S. Maheswaran y DA Haber, "Un conducto a la metástasis: biología celular tumoral circulante", Genes \& Development, vol. 31, no. 18, págs. 1827-1840, 2017.Ver en: Sitio del editor | Google Académico

40. TJ Li, YM Jiang, YF Hu et al., "Los neutrófilos productores de interleucina-17 vinculan los estímulos inflamatorios con la progresión de la enfermedad a promover la angiogénesis en el cáncer gástrico", Clinical Cancer Research , vol. 23, no. 6, págs. 1575-1585, 2017.Ver en: Sitio del editor | Google Académico

41. NE Burr, MA Hull y V. Subramanian, "¿El uso de medicamentos antiinflamatorio no esteroideos con aspirina o sin aspirina previene el cáncer colorrectal en la enfermedad inflamatoria intestinal?" World Journal of Gastroenterology, vol. 22, no. 13, págs. 3679-3686, 2016.Ver en: Sitio del editor |Google Académico

42. J. Pillay, I. den Braber, N. Vrisekoop et al., "El marcado in vivo con $2 \mathrm{H} 2 \mathrm{O}$ revela una vida útil de neutrófilos humanos de 5,4 días" , Blood, vol. 116, no. 4, págs. 625-627, 2010.Ver en: Sitio del editor | Google Académico

43. W. Liang y N. Ferrara, "El papel complejo de los neutrófilos en la angiogénesis y metástasis tumorales", Cancer Immunology Research , vol. 4, no. 2, págs. 83-91, 2016.Ver en: Sitio del editor |Google Académico

44. A. Swierczak, KA Mouchemore, JA Hamilton y RL Anderson, "Neutrófilos: contribuyentes importantes para la progresión tumoral y la metástasis", Cancer Metastasis Reviews, vol. 34, no. 4, págs. 735-751, 2015.Ver en: sitio del editor Google Académico

45. R. Ogiya, N. Niikura, N. Kumaki et al., "Comparación de linfocitos infiltrantes de tumores entre tumores primarios y metastásicos en pacientes con cáncer de mama", Cancer Science, vol. 107, no. 12, págs. 1730-1735, 2016.Ver en: sitio del editor Google Académico

46. Fang T, Wang Y, Yin X, Zhai Z, Zhang Y, Yang Y, You Q, Li Z, Ma Y, Li C, Song H, Shi H, Zhang Y, Yu X, Gao H, Sun Y, Xie R, Xue Y. Diagnostic Sensitivity of NLR and PLR in Early Diagnosis of Gastric Cancer. J Immunol Res. 2020 Mar 7;2020:9146042. doi: 10.1155/2020/9146042. PMID: 32211444; PMCID: PMC7081040. 